\title{
Underwater Acoustic Communications Performance Modeling in Support of Ad Hoc Network Design
}

\author{
Warren L. J. Fox and Payman Arabshahi \\ Applied Physics Laboratory \\ University of Washington \\ 1013 N.E. 40th St. \\ Seattle, WA 98105-6698 \\ Email: \{warren,payman\}@apl.washington.edu
}

\author{
Sumit Roy and Nathan Parrish \\ Department of Electrical Engineering \\ University of Washington \\ Campus Box 352500 \\ Seattle, WA 98195-2500 \\ Email: \{sroy,nparrish\}@u.washington.edu
}

\begin{abstract}
This paper discusses a methodology for predicting underwater acoustic communications performance using high fidelity acoustic time series simulation and acoustic modem processing emulation. Multiple source/receiver combinations can be simultaneously simulated, so that aspects of a complete underwater network can be studied. Here, the fundamental modeling and emulation capability will be described, with examples of the propagation modeling, time series simulation, and modem processing over multiple realizations of example communications channels. The results show the dependence of source and receiver location in the water column with respect to the sound speed profile on communications performance. The utility of such simulations for ad hoc network design in the presence of moving communications nodes will be discussed.
\end{abstract}

\section{INTRODUCTION}

As the use of groups of underwater vehicles (often in concert with a subset of fixed underwater nodes) proliferates in a wide variety of applications, the need for robust underwater acoustic communications networks also increases. The difficulties of the underwater acoustic communications channel are well-known: overall bandwidth is limited due to acoustic absorption that increases with frequency, and typical shallow water regions where communications are desired have high degrees of spatial and temporal variability.

Designing an underwater network is often considered a constraint imposed on other system optimization considerations whose metrics are derived from the intended mission of the sensing nodes. Therefore, nodes should be deployed such that the overall sensing mission will be successful, but mission success may also depend on information being passed between nodes through the communications network. The networking schemes will likely be ad hoc in nature if moving vehicles comprise some set of the nodes. Although specific requirements may differ among applications, one fundamental requirement is usually some basic level of connectivity between nodes. Successful design of a network configuration then depends on the ability to predict the likelihood of communications packets being successfully received.

This paper will describe a methodology for modeling acoustic communications performance based on high-fidelity acoustic time series modeling and acoustic modem processing emulation. The research project under which this work has taken place is utilizing the Micro-Modem developed at the Woods Hole Oceanographic Institution (WHOI) [1]. A software package that emulates the transmit signal generation and receive signal processing and demodulation process of the Micro-Modem has kindly been provided to the authors by WHOI researchers. In order to accurately model the effects of acoustic propagation through the ocean between potentially moving sources and receivers, the Sonar Simulation Toolset (SST) [2] is being used.

SST allows a user to specify an ocean environment with a wide variety of parameters relevant to acoustic signal propagation and reception: sound speed profile, bathymetry, surface/bottom characteristics, ambient noise levels, etc. The user can also specify locations and trajectories of acoustic sources and receivers within that environment, and signals to be transmitted by the sources. SST then uses acoustic propagation models and time series simulation techniques to produce properly calibrated digital time series of the signals that would be "heard" by the receivers. These time series can then be operated on by signal processing algorithms, such as the Micro-Modem emulator mentioned above.

Section II of this paper will further describe the acoustic and time series modeling procedures, and show examples of simulated received acoustic communications time series. Section III will show several examples of using the modem emulation software to process the received signals in order to estimate modem performance. Section IV will discuss how these capabilities can be used in ad hoc underwater acoustic network design, and other factors that need to be considered in the design of such systems. Section V will summarize the results and comment on future work.

\section{Acoustic And Time Series Modeling}

The field of acoustic propagation modeling is broad and diverse, with several different numerical approximations to solving the wave equation available depending on the specifics of the application [3]. Considering the relatively high frequencies typically used for underwater acoustic communications (e.g., the WHOI Micro-Modem operates at frequencies from roughly $7.5 \mathrm{kHz}$ to $30 \mathrm{kHz}$ ), ray tracing approaches should provide adequate levels of fidelity for predicting received 


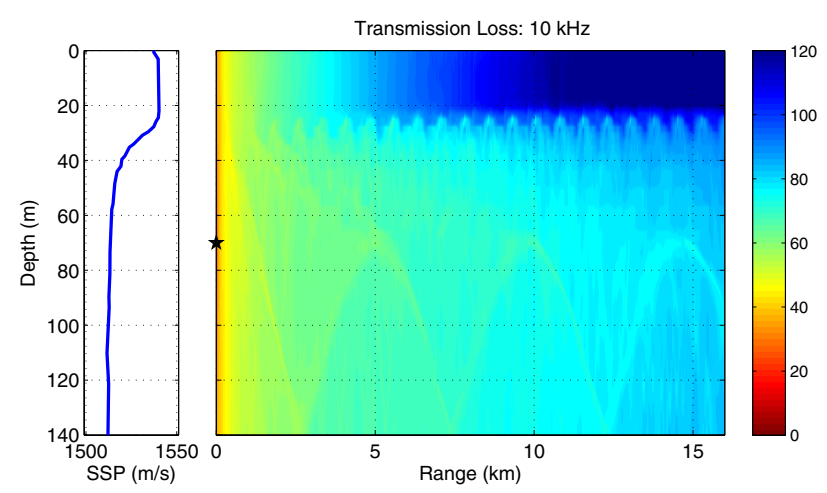

Fig. 1. Transmission loss $(\mathrm{dB})$ at $10 \mathrm{kHz}$ for a source at $70 \mathrm{~m}$ depth.

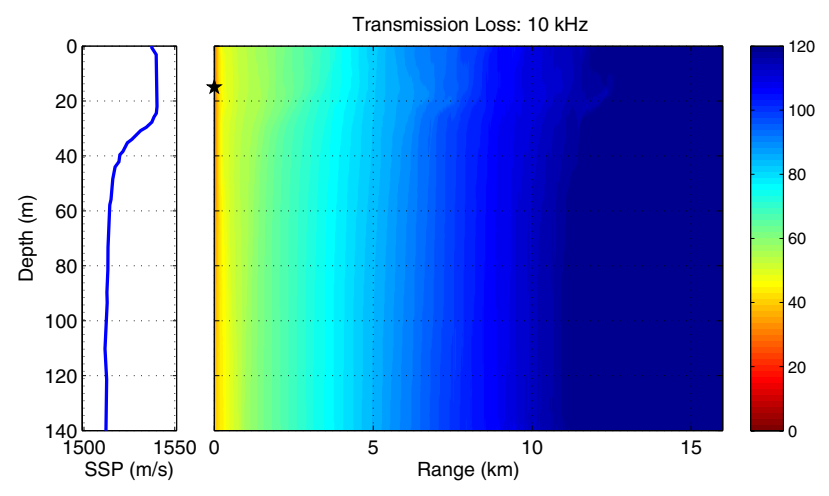

Fig. 2. Transmission loss $(\mathrm{dB})$ at $10 \mathrm{kHz}$ for a source at $15 \mathrm{~m}$ depth.

signal levels and arrival structure. Specifically, we use the Gaussian ray bundle approach [4].

Figure 1 shows an example of acoustic propagation modeling in a two-dimensional vertical slice of the water column computed using ray tracing methods. The range-independent sound speed as a function of depth used for this calculation is plotted in the left pane. The right pane shows the transmission loss (TL) as a function of range and depth due to a steady state $10 \mathrm{kHz}$ source located at a depth of $70 \mathrm{~m}$. Transmission loss is usually stated in $\mathrm{dB}$, and describes the weakening of sound at points distant from an acoustic source due to spreading (waveguide propagation) and absorption [5]. Note that this example exhibits high values of TL above the thermocline (i.e., above $30 \mathrm{~m}$, where the sound speed profile changes quickly with depth), meaning that low levels of acoustic energy are propagating into this region. Note also that somewhat complicated patterns of propagating energy appear further out in range below the thermocline due to downward refracting conditions and multiple interactions with the sea bottom interface.

Figure 2 shows a similar TL calculation as in Fig. 1, except the source is now at a depth of $15 \mathrm{~m}$. The resulting TL pattern in range and depth is dramatically different in this example. Due to a slight concavity in the sound speed profile near the source, there is a divergence of propagating energy, creating a situation where acoustic energy is not propagated well either above or below the thermocline.

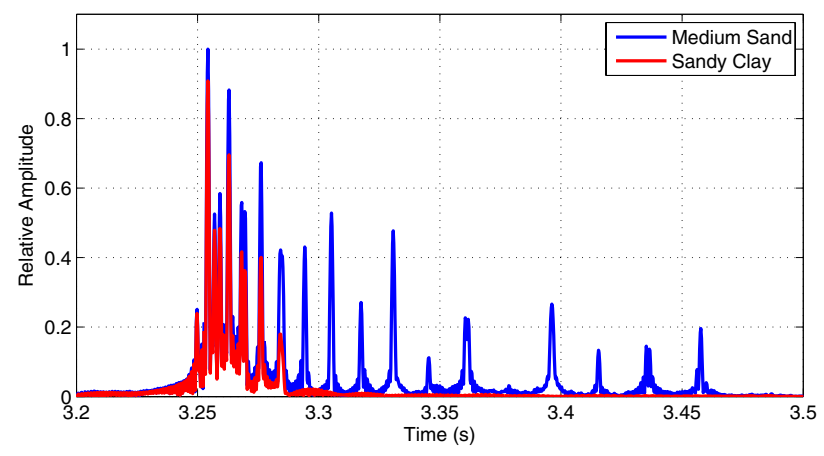

Fig. 3. Channel impulse responses vs. bottom type.

Such transmission loss calculations are useful for estimating levels of propagating energy in the water column. However, for testing signal processing algorithms, a simulation of the acoustic time series that would arrive at a hydrophone is required. As mentioned in the Introduction, the Sonar Simulation Toolset (SST) is a computer program that can generate such simulated time series. The development roots of SST at the Applied Physics Laboratory, University of Washington can be traced back to acoustic time series simulation work started over 30 years ago [2]. SST is specifically designed to generate such acoustic time series, and its development path has seen a consistent trend of extending functionality and increasing simulation fidelity.

For underwater acoustic communications applications, the "Direct" simulation mode of SST is employed. In this mode, one or more sound emitters are specified, and the properties of the source(s), receiver(s), and propagation model are combined and reduced to a set of filters and delays that are applied to emitted signals to produce simulated signals at one or more receivers. SST uses the same fundamental eigenray results used to compute TL at a given location in the ocean to produce this set of filters. SST can also generate reverberation and target echoes for active sonar applications. Ambient noise with a specified power level and spectrum can be simulated within SST and added to received signals.

Figure 3 shows an example of such time series simulations. A scenario similar to that seen in Fig. 1 was simulated, with source and receiver below the thermocline, $5 \mathrm{~km}$ apart. A $1 \mathrm{kHz}$ bandwidth, $0.5 \mathrm{~s}$ long linear FM chirp signal was transmitted and the signal at the receiver was generated for two different bottom types, one defined as "Medium Sand," and the other as "Sandy Clay." The geophysical parameters for these canonical bottom types are described in reference [6], but for this work it will suffice to describe the "Sandy Clay" bottom as much softer, with higher levels of forward loss vs. grazing angle associated with each bottom interaction than the "Medium Sand" case. The received signals were matched filtered with the transmitted signal, and the effective "channnel impulse responses" (CIRs) between the chosen source and receiver locations for these two bottom types are plotted in Fig. 3. 


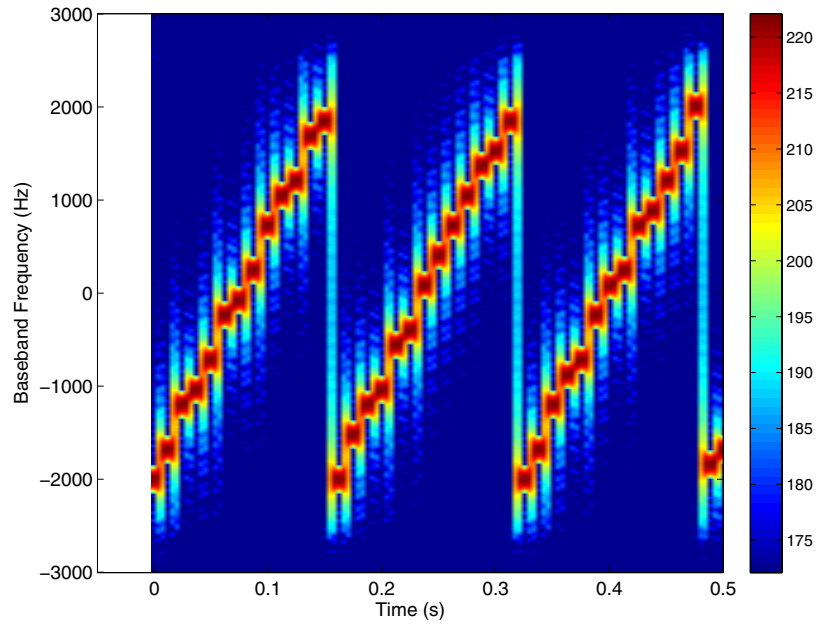

Fig. 4. Spectrogram of transmitted FH-FSK signal.

In Fig. 3, it can be seen how more energy is transmitted into the softer bottom type with each bottom interaction, significantly shortening the effective CIR compared to the harder bottom type. Such time spreading can cause intersymbol interference (ISI) in communications systems, therefore it is important to be able to accurately model such effects in a variety of environments in order to study acoustic modem processing algorithms. SST allows variation of many parameters of the environment in order to perform such studies: sound speed profile, bathymetry, bottom type, and surface conditions, as well as ambient noise levels. It also allows wide latitude in specification of source and receiver locations and trajectories, so that the effects of location in the water column and Doppler shifting can be studied.

Figure 4 shows an example of a typical transmit signal generated by the WHOI Micro-Modem [1]. The figure shows a spectrogram of the first 0.5 seconds of the FHFSK (Frequency-Hopped Frequency-Shift Keying) signaling waveform at baseband. The true center frequency for this example is $9680 \mathrm{~Hz}$, but a baseband representation is used to take advantage of processing efficiencies in SST. This signaling scheme allows a data rate of 80 symbols per second. Note that the frequency hopping nature of the signal provides for $150 \mathrm{~ms}$ of channel clearing time until that frequency band is potentially reused for another symbol.

Figure 5 shows the results of using the signal from Fig. 4 as the transmit signal in a scenario similar to that of Figs. 1 and 3. Figure 5 is a baseband spectrogram of the received signal with the same dynamic range in the color scale as Fig. 4. Some of the effects of the propagation simulation are immediately clear. One is the time spreading due to multipath propagation that appears in the time dimension after each frequency chip. Also, frequency-selective fading is apparent at some frequencies due to closely spaced multipath arrivals that destructively interfere at those frequencies. Ambient noise has been added to the signal, and can be observed by the elevated background levels, especially noticeable in the upper lefthand portion of the

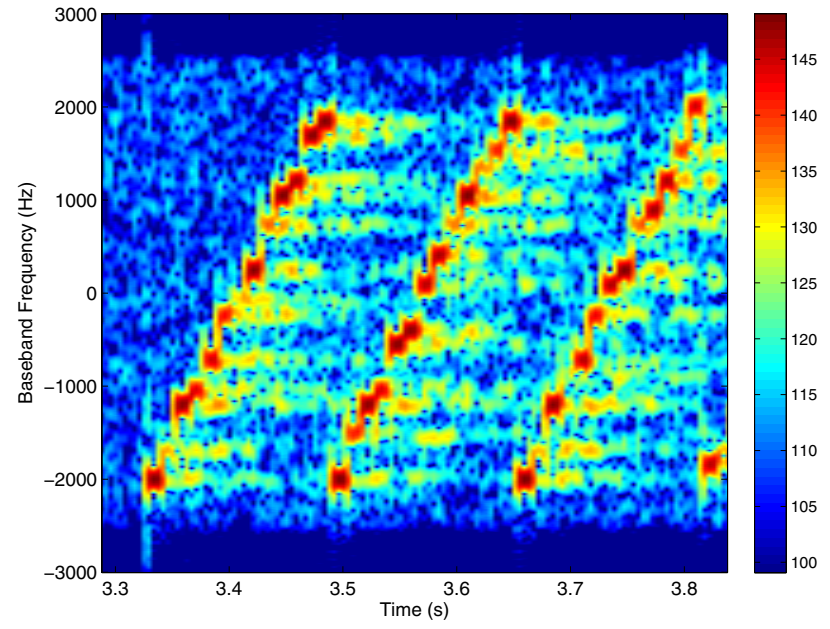

Fig. 5. Spectrogram of received FH-FSK signal.

spectrogram (before any signal has arrived in those frequency bands). Section III will demonstrate how such simulations can be used to study modem performance in concert with modem processing emulation software.

\section{Emulated Modem Processing}

As shown in the previous section, SST can generate simulated received waveforms that incorporate much of the physics apparent when an acoustic signal is propagated through the ocean. These signals can then be operated on by signal processing algorithms. As mentioned in the Introduction, a software package that emulates the processing of the WHOI Micro-Modem has been provided to the authors, and can be used to examine Micro-Modem performance in simulated environments.

In the examples to be shown in this Section, communications signal acquisition has been simulated by prepending a 20 ms long linear FM waveform (and a quiescent gap to allow channel clearing) to the signaling waveform generated by the WHOI software. The LFM signal covers the same bandwidth as the signaling waveform, and has the same peak amplitude. The simulation is run such that the bulk travel time is estimated from known geometry, and a one second lead buffer is included in the simulated reception. A matched filtering operation is then performed on the total received sequence, and the maximum of the matched filter output is considered to be the arrival time of the leading edge of the LFM acquisition signal. The communications sequence is then obtained based on the known delay between the LFM and the signaling waveform, and processed by the WHOI MicroModem emulation software. This is known to be an imperfect emulation of the acquisition processing of the Micro-Modem, but should give some indication of performance issues related to missed acquisition. In fact, there is some indication that there may be cases where there is enough signal-to-noise ratio (SNR) for communications to be effective, but failed acquisition results in packet loss. 


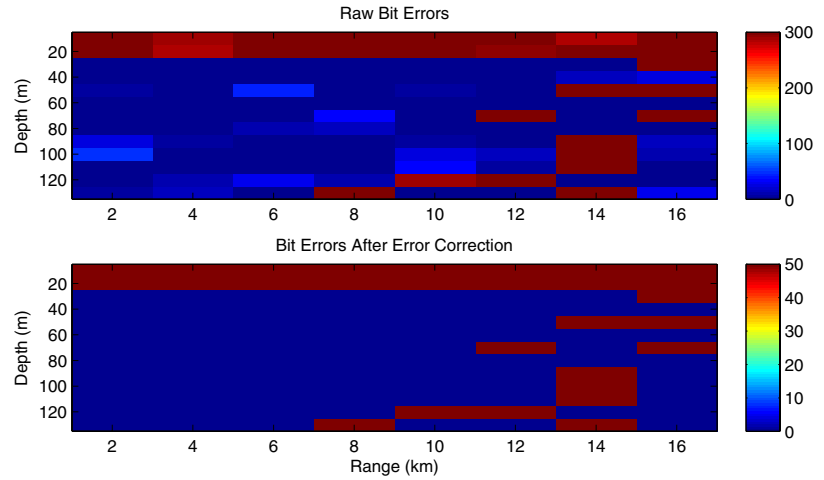

Fig. 6. Modem performance (single realization) as a function of receiver location for scenario of Fig. 1.

One way to assess modem performance in a given scenario is to place a transmitting modem at a certain depth, create a grid of hypothetical modem receivers in the water column, simulate the propagation of the signal to all receivers, and process the received signals as described above. When doing this for the scenario pictured in Fig. 1, the results can be displayed as shown in Fig. 6. Modem receivers were simulated every $10 \mathrm{~m}$ in depth and every $2 \mathrm{~km}$ in range. The top panel of Fig. 6 shows the raw number of bit errors as a function of receiver position. The bottom panel shows the number of bit errors after the built-in error correction of the Micro-Modem is applied. Blue regions in the lower panel indicate successful communications packet transmittal.

As can be seen in Fig. 6, there is no communications performance to receiving modems above the thermocline, which is consistent with the high levels of TL that would be observed there. Also, just as there are complicated patterns in the TL at further ranges below the thermocline, there is spatially variable modem performance in the same region.

Figure 6 shows the results for a single realization of the modeled propagation, but it may be desireable to examine the results for a statistical ensemble of model runs. Figures 7 and 8 show the mean and standard deviation, respectively, for the scenario of Fig. 6 over 30 realizations. Note that the single realization case and the mean of the ensemble agree in general terms, although there are some receiver positions in the single realization case that appear to be anomalous when the ensemble is considered. In the standard deviation plot, we notice that there is low variability above the thermocline (uniformly bad performance), but that there are regions of high performance variability below the thermocline and at longer ranges. An analysis like this would likely lead a network designer to define a performance "envelope" in depth and range for locations of receivers (for this particular transmitter depth) at which communications performance would be robust.

A similar experiment can be run for the case of a transmitter at $15 \mathrm{~m}$ depth in the same environment (as in the TL case of Fig. 2), and the results for this case are shown in Figures 9 and 10 (mean and standard deviation, respectively, over 30 realizations). Consistent with the TL results for this config-

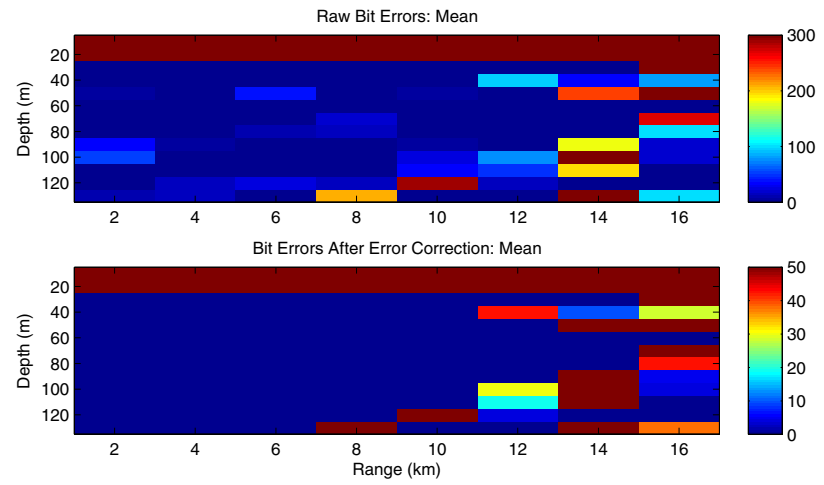

Fig. 7. Average modem performance over 30 realizations as a function of receiver location for scenario of Fig. 1.

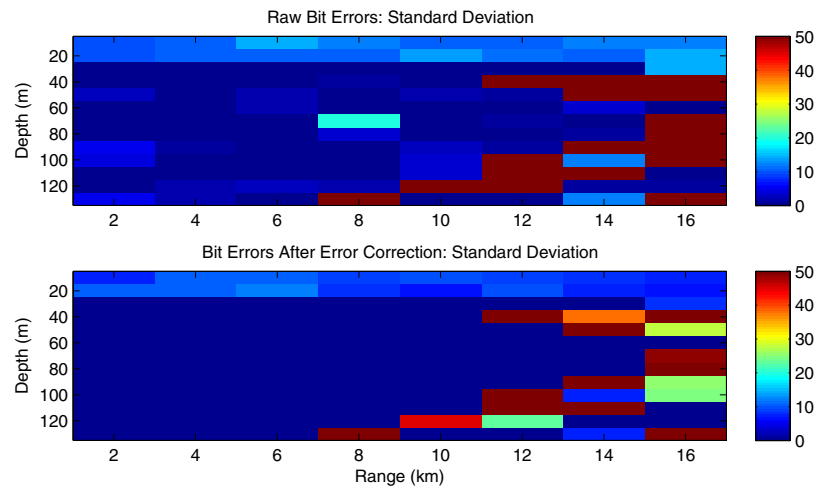

Fig. 8. Standard deviation of modem performance over 30 realizations as a function of receiver location for scenario of Fig. 1.

uration, very poor communications performance can be seen at nearly all receiver locations. It appears that there may be some isolated ray paths that provide propagation good enough for the modem processing to utilize, but it is not clear that these would be robust signals that could be relied on in the real ocean given mostly poor performance in adjoining cells.

\section{Ad Hoc Networking}

Undersea acoustic networks can be composed of entirely fixed nodes, entirely mobile nodes, or a mixture of the two. Such networks typically need to be $a d$ hoc in nature, either because communicating nodes are moving with respect to each other or because acoustic conditions can change with time. Mobile undersea platforms and fixed nodes are usually deployed with some sensing mission in mind, such as making physical measurements of ocean properties or acoustic surveillance. These mission objectives will be the main driver in determining how nodes are placed or moved within the environment.

Communications requirements within such collections of sensing nodes will differ depending on the application, and will provide additional requirements or constraints on the main sensing mission. If the sensing missions of the various nodes are loosely coupled, there may be only the requirement for data to be transmitted out of the network and back to shore-based 


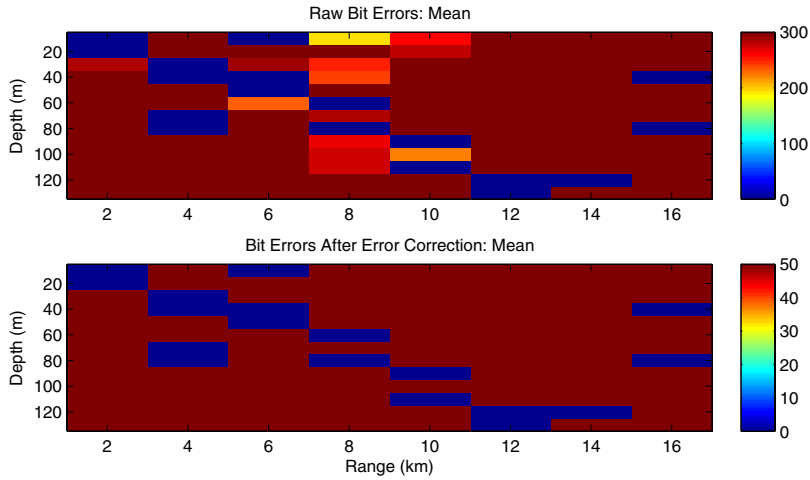

Fig. 9. Average modem performance over 30 realizations as a function of receiver location for scenario of Fig. 2.

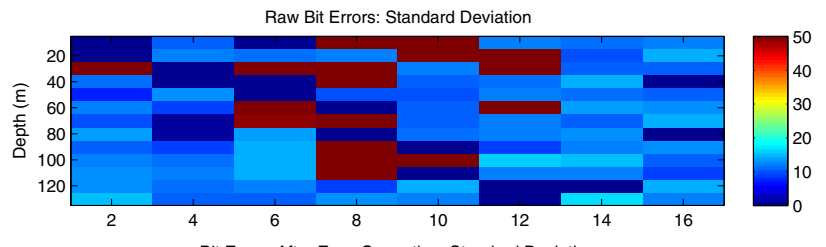

Bit Errors After Error Correction: Standard Deviation

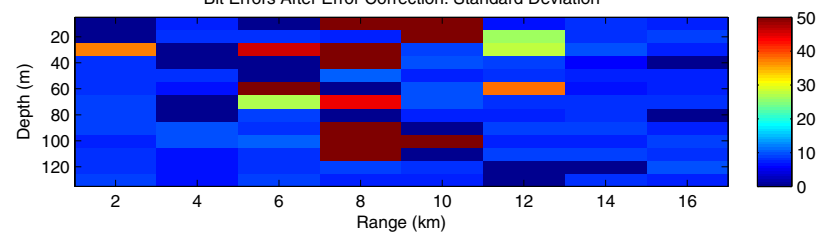

Fig. 10. Standard deviation of modem performance over 30 realizations as a function of receiver location for scenario of Fig. 2 .

analysis centers, with minimal requirements for inter-node connectivity. If the mission is highly collaborative in nature, the communications requirements may be more stringent, such that all nodes must have constant communications capability with at least one other node such that information can be passed around the network and all nodes can be fully informed.

For applications where high degrees of inter-node connectivity are required, analyses such as those found in the previous Section would be extremely useful. Consider the environment studied in Section III. Although more source depths would need to be analyzed, it appears that robust communications between two modems below the thermocline would be possible out to ranges of $10 \mathrm{~km}$ or so (at the $\sim 10 \mathrm{kHz}$ operating frequency analyzed). It does not appear that communications would be possible between modems where one is above the thermocline and the other below it. This restriction could have a profound effect on the concept of operations for various sensing missions. If the sensors are required to make measurements above the thermocline, the platforms would then need to move deeper in order to transmit information to other nodes below the thermocline.

The ultimate vision of how such a time series simulation and modem performance emulation approach would work is in a completely closed-loop system. As the sensing platforms move through the water column, they could be measuring sound speed as a function of depth, which is one of the key drivers to predicting sound propagation and communications performance. As this data is transmitted out of the network and analyzed, updated communications performance envelopes could be computed and transmitted back to the field of nodes, either in the form of explicit positioning instructions due to a centralized controller, or in more raw form such that the mobile nodes can self-organize given the communications performance information.

\section{CONCLUSION}

This paper has outlined a methodology for assessing underwater acoustic communications performance in support of $\mathrm{ad}$ hoc network design. By using a combination of high fidelity acoustic time series simulation and acoustic modem signal processing emulation, the communications performance between a transmitting modem at one location and hypothetical receiving modems at a number of other locations can be assessed. The analysis can be repeated as necessary for other transmitter locations, and for different environmental variables. It was shown how statistical ensembles can be generated in order to assess variability of modem performance. It was also discussed how this capability could be embedded in a closedloop system for maintaining connectivity between all nodes of an undersea sensing network.

One immediate item of future work is validation of these modeling procedures against measured data. The authors have access to several data sets where packet success rate between Micro-Modems on moving vehicles was measured. After reconstruction of the environmental inputs and platform trajectories, time series simulations and modem emulations can be run to compare the measured and modeled performance.

\section{ACKNOWLEDGMENTS}

This work was supported by the NASA Earth Science Technology Office's Advanced Information Systems Technology (AIST) Program under award number AIST-05-0030. The authors would like to thank Mr. Lee Freitag of WHOI for providing the Micro-Modem emulation software.

\section{REFERENCES}

[1] L. Freitag, M. Grund, S. Singh, J. Partan, P. Koski, and K. Ball, "The WHOI micro-modem: An acoustic communications and navigation system for multiple platforms," in Proc. MTS/IEEE Oceans 2005, vol. 2, Sep. 2005, pp. 1086-92.

[2] R. P. Goddard, "The Sonar Simulation Toolset, Release 4.1: Science, Mathematics, and Algorithms," Applied Physics Laboratory, University of Washington, Tech. Rep. APL-UW TR 0404, Mar. 2005.

[3] F. B. Jensen, W. A. Kuperman, M. B. Porter, and H. Schmidt, Computational Ocean Acoustics. New York: AIP Press, 1994.

[4] H. Weinberg and R. E. Keenan, "Gaussian ray bundles for modeling highfrequency propagation loss under shallow-water conditions," J. Acoust. Soc. Am., vol. 100, no. 3, pp. 1421-31, 1996.

[5] R. J. Urick, Principles of Underwater Sound, 3rd ed. New York: McGraw Hill, 1983.

[6] "APL-UW High-Frequency Ocean Environmental Acoustic Models Handbook," Applied Physics Laboratory, University of Washington, Tech. Rep. APL-UW TR 9407, Oct. 1994. 